\title{
Measurement of thermal conductivity in situ in mixed materials, e.g. soils
}

\author{
A. R. P. JANSE and G. BOREL \\ Laboratory of Soils and Fertilizers, State Agricultural University, and \\ Service Institute for Applied Mechanics and Technical Physics in \\ Agriculture respectively, Wageningen, Netherlands
}

\section{Summary}

The general theory of heat transfer is presented, together with a method for the determination of thermal conductivity.

The practical field apparatus is described and suggestions are made for its use.

\section{Introduction}

In the description of heat transfer in any medium, two specific properties are involved which should be considered independently, viz. heat capacity (c) and thermal conductivity $(\lambda)$. Many formulae deal with their ratio, i.e. thermal diffusivity a $\left(=\frac{\lambda}{c \varrho}\right)$. A detailed and up-to-date review of soil-thermal properties and possibilities of calculating them is discussed by DE VRIES (1952).

In the present article an elementary introduction will be given, with special reference to the determination of thermal conductivity.

As a static property, heat capacity $\left(\mathrm{cal} \mathrm{cm}^{-3}{ }^{\circ} \mathrm{C}^{-1}\right)$ is defined as the number of calories required to raise the temperature of one cubic centimetre of the material concerned by one degree centigrade. In the case of a soil, this is equal to the sum of the relative amounts of the components multiplied by their respective specific-heat values. Thus,

$$
\mathrm{C}=\Sigma_{\mathrm{i}} \mathrm{x}_{\mathrm{i}} \varrho_{\mathrm{i}} \mathrm{s}_{\mathrm{i}}
$$

in which $\mathrm{C}=$ heat capacity $\left(\mathrm{cal} \mathrm{cm}-3{ }^{\circ} \mathrm{C}^{-1}\right), \mathrm{x}_{\mathrm{i}}=$ volume fraction of each component $i$ in one cubic centimetre, $\varrho_{i}=$ density $\left(\mathrm{g} \mathrm{cm}^{-3}\right), s_{\mathrm{i}}=$ specific heat of component $\mathrm{i}$ in cal $\mathrm{g}^{-1}{ }^{\circ} \mathrm{C}^{-1}$. With the aid of this formula and the data given in the table, and provided the phase distribution is known, the heat capacity of a soil can be calculated sufficiently accurately for most practical purposes. The influence of water on the heat capacity of a soil can be readily deduced from this table. It should be emphasised that the arrangement of the given components has no effect on the value of $\mathbf{C}$ in mixed materials.

The dynamic heat properties of a soil are the factors that determine the flow of heat in soils. The thermal conductivity $\lambda$ is the most important factor. It is defined as the heat flux in cal per second, per $\mathrm{cm}^{2}$, per unit of temperature gradient. Con-

Received for publication 24th July, 1964.

Neth. J. agric. Sci., Vol. 13 (1965) No. 1 (February) 
sequently, the thermal conductivity of a soil is mainly determined by the number and the surface areas of contact zones between soil particles, mainly quartz. The calculation is complicated by the number of contact zones, is greatly influenced by the spatial arrangement of the components, resulting in a number of contact surfaces. It can be directly inferred from the TABLE that an increase in moisture content causes an increase in the thermal conductivity.

TABLE

$\begin{array}{lcc} & \mathrm{S}\left(\mathrm{cal} \mathrm{g-1}{ }^{\circ} \mathrm{C}-1\right) & \lambda\left(\mathrm{cal} \mathrm{cm-1}{ }^{\circ} \mathrm{C}-1 \mathrm{10}-3\right) \\ \mathrm{H}_{2} \mathrm{O} \ldots \ldots \ldots \ldots \ldots \ldots \ldots \ldots & 1 & 1.42 \\ \text { Air (saturated with water) } & 0.28 & 0.24 \\ \text { Quartz } \ldots \ldots \ldots \ldots \ldots \ldots \ldots \ldots & 0.19 & 20.7 \\ \text { Humus } \ldots \ldots \ldots \ldots \ldots \ldots \ldots & 0.47 & -\end{array}$

In the method to be described thermal conductivity is determined from variations in temperature close to a heat source. This method is dynamic, which makes it useful for determinations in soils, and, due to its rapidity, transport of water is prevented during the determination. In practice, excessive heating can be prevented when the range of temperature variations does not exceed $2^{\circ} \mathrm{C}$. Under normal conditions the measurements are independent of the initial temperature. In the laboratory, an accuracy of $2 \%$ can be obtained. Use is made of a needle in which a heating filament and a thermocouple are built. The reference junction of the thermocouple can be placed in any suitable medium, since only relative temperature variations are to be measured.

The method is based on the equation for cylindrial flow. This equation can be derived from basic heat-flow equations, given in uni-dimensional form:

a. The diffusion equation: $F=-\lambda \frac{\mathrm{d} \Theta}{\mathrm{dx}}$,

in which $\mathbf{F}=$ the heat flux per $\mathrm{cm}^{2}$ en $\Theta=$ temperature.

b. The equation of continuity: $\frac{\mathrm{dW}}{\mathrm{dt}}=-\frac{\mathrm{dF}}{\mathrm{dx}}$,

in which $\mathrm{W}=$ the heat content per $\mathrm{cm}^{3}$ of the material,

$\mathrm{x}=$ unit length in the direction of flow,

$\mathbf{t}=$ time in sec.

c. The relationship between heat content and temperature: $\frac{d W}{d t}=C \frac{d \Theta}{d t}$,

in which $\mathrm{C}=$ heat capacity of the soil (cal $\left.\mathrm{cm}^{-3}{ }^{\circ} \mathrm{C}^{-1}\right)$.

d. Combining the equations, we obtain: $\frac{\mathrm{d} \Theta}{\mathrm{dt}}=\frac{\lambda}{\mathrm{c}} \frac{\mathrm{d}^{2} \Theta}{\mathrm{dx}^{2}}$.

In the cylindrical case, one may choose as an acceptable solution of the Fourier differential equation:

$$
\Theta_{2}-\Theta_{1}=\frac{\mathrm{q}}{4 \pi \lambda} \ln \frac{\mathrm{t}_{2}}{\mathrm{t}_{1}},
$$

in which $\Theta_{2}-\Theta_{1}$ denotes the increase in temperature near the heating source,

$t_{2}-t_{1}$ the time period during which the temperature variation was measured,

q the total amount of heat developed. 
The solution can only be used provided certain precautions are taken. Thus for instance, time zero cannot be used, since the capillary itself requires time for heating up. The length of this source, which strictly speaking is not infinite, is a further problem. Apparatus could therefore only be designed after considerating the way in which these problems affected the measuring results from a theoretical point of view. A good treatment is presented in van Drunes's (1949) dissertation.

Finally the values for heat developed and measured temperature variations should be known.

Since the resistance of the wire is known, the amount of heat $\mathrm{q}=0.24 \mathrm{i}^{2} \mathrm{Rt}$, in which $\mathrm{i}=$ the electrical current in Ampères, $\mathrm{R}=$ resistance of the heating wire in Ohms and $\mathrm{t}=$ time in seconds.

Plotting the increase of temperature log. time will result in a straight line. The slope of this line represents $q / 4 \pi \lambda$ from which $\lambda$ can be calculated.

\section{Apparatus}

The apparatus essentially consists of three units, viz. the needle, a current supply and a microvoltmeter. The apparatus is built in a wooden box with a handle, so that it can easily be carried in the field (FIG. 1). The needle is an ordinary hypodermic needle (FIG. 2) with an outside diameter of $1 \mathrm{~mm}$ and a length of $20 \mathrm{~cm}$, which makes its length practically infinite compared to its thickness. The inner diameter of the needle is $0.6 \mathrm{~mm}$ and in it are placed a heating wire of $0.1 \mathrm{~mm}$ thickness in the form of a loop with an electrical resistance of $28 \Omega$, together with a copper-constantan thermocouple of $0.15 \mathrm{~mm}$ thickness, the two being insulated from each other. The needle is filled with resin in vacuo and connected to stronger wires at the upper end of the inside wiring. A special probe has been designed for measurements to a depth of $45 \mathrm{~cm}$ (FIG. 2). The probe is first driven into the soil, after which the needle can be lowered through this protecting probe.

The heating wire is connected to the constant current supply situated in the righthand section of the instrument box (FIG. 1) (LIEN, 1962). This supply delivers an adjustable and stabilized current varying from 0.1 to $0.8 \mathrm{~A}$, depending on external conditions (FIG. 3). The current is regulated by transistor $T_{1}$, which base current is determined by the output of the transistorized differential amplifier. This amplifier compares at its input the voltage over the standard resistance $\mathbf{R}$, (which is proportional to the current $\mathrm{i}$ ) with an adjusted fixed voltage originating from the stabilized input voltage.

The input voltage of the amplifier must be zero. If the current $i$ increases, the input terminal I of the amplifier becomes positive with regard to terminal II and the transistor $T_{1}$ becomes less conductive, until the current again reaches its adjusted value. If the current decreases, terminal II of the amplifier becomes positive with regard to $I$, and $T_{1}$ becomes more conductive.

The power can be obtained in the field from two 12-volt car batteries; for laboratory use a stabilized 24-volt supply is built in that can be connected to the mains. The regulating transistor $\mathrm{T}_{2}$ (FIG. 4) is again controlled by a transistorized differential amplifier that compares the output voltage with a reference voltage of 24 volts from a zener diode. The stability of the output voltage is $0.5 \%$ for $10 \%$ mains voltage variations.

The EMF of the thermocouple is measured with the microvoltmeter (Fig. 5) (MIDDLEBROOK and TAYLOR, 1961). The circuit is a cascade connection of two differential 
FIG. 3. Current supply

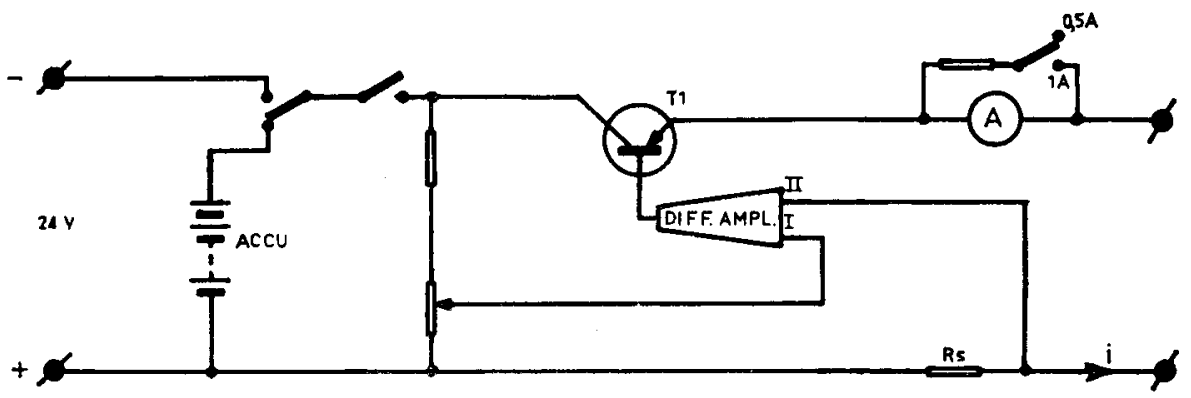

FIG. 4. Voltage supply

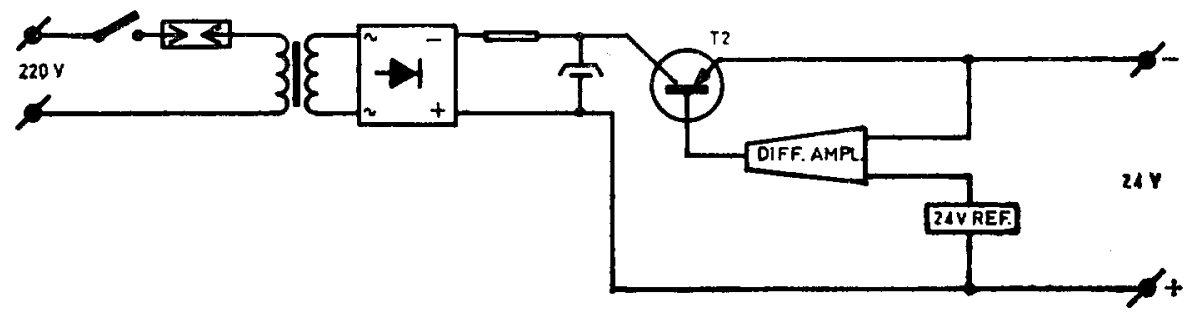

amplifiers (FIG. 6) and operates on a 9 V D.C. dry battery. Before measuring, the meter input must be balanced and the pointer set at zero by pressing down the balance-zero button and adjusting the controls involved. In a later version the sensitivity was increased over the ranges shown in the picture. One can now select four ranges giving full-scale deflections for $100 \mu \mathrm{V}, 250 \mu \mathrm{V}, 1 \mathrm{mV}$ and $2.5 \mathrm{mV}$. For a

FIG. 6. Microvoltmeter

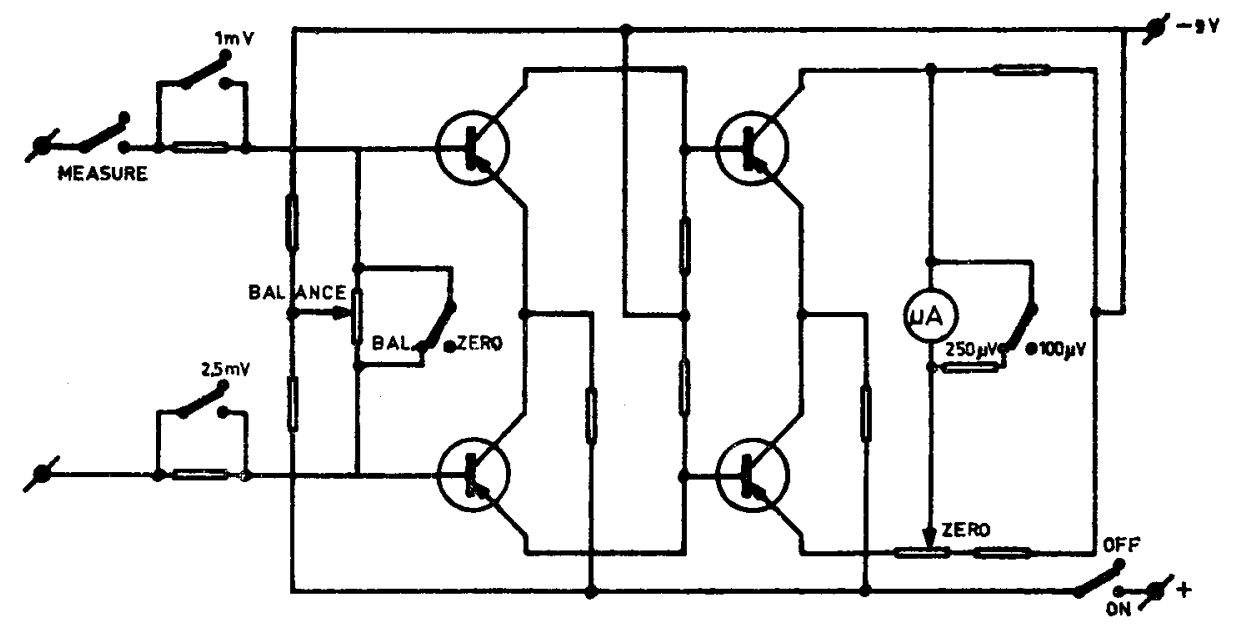




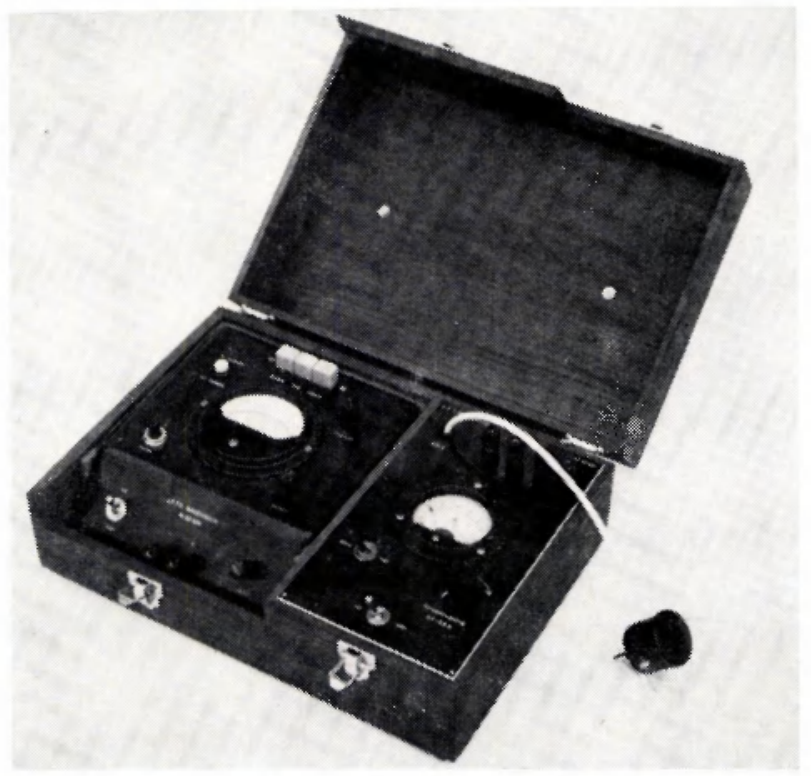

FIG. 1

Complete apparatus for measurement of thermal conductivity in the field

FIG. 2. Needle with heating wire and thermocouple inside in protecting probe for measurement at greater depths (centre), handle (top right) and electric plug (top left) 20-cm needle (bottom left)

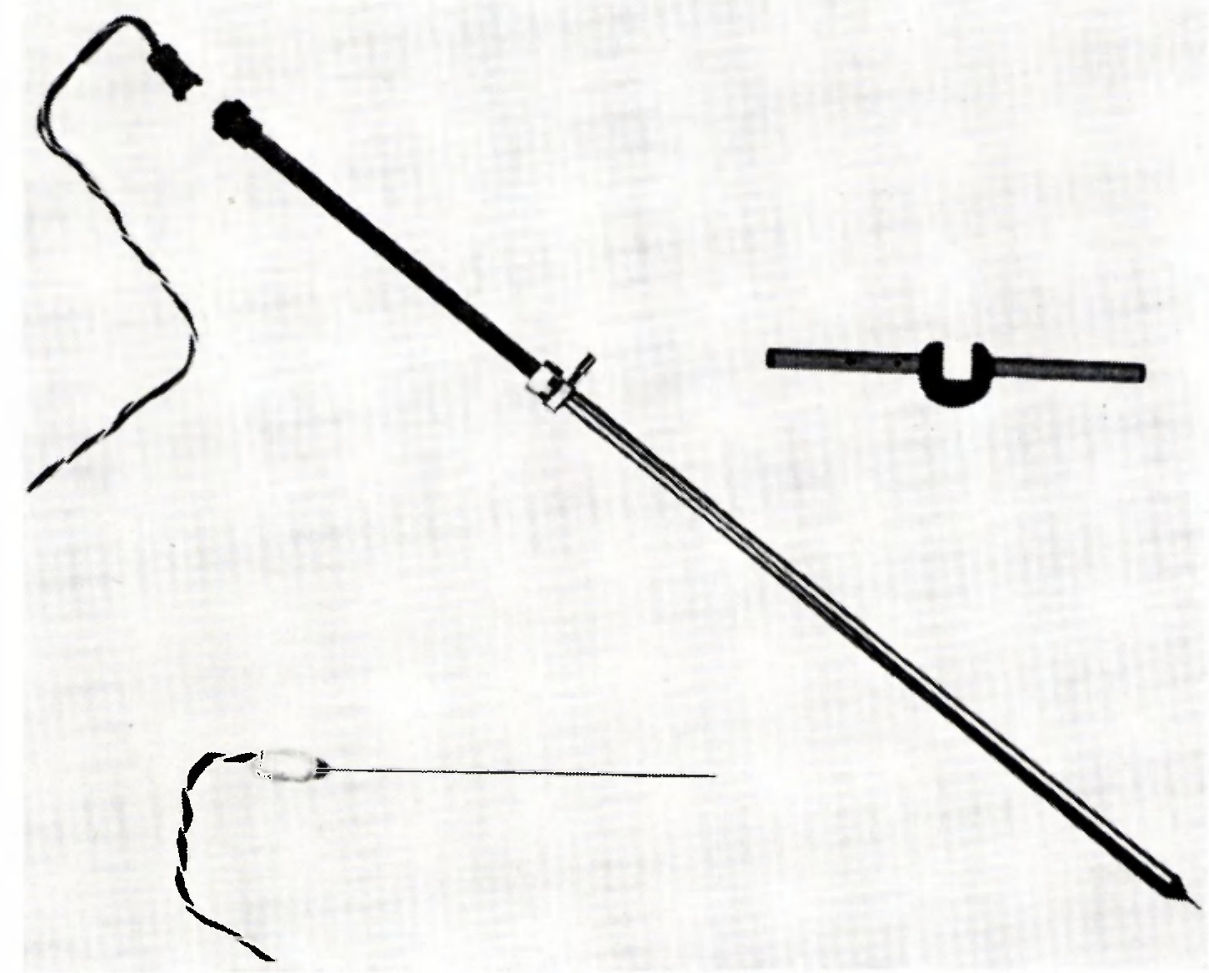


FIG. 5. Microvoltmeter

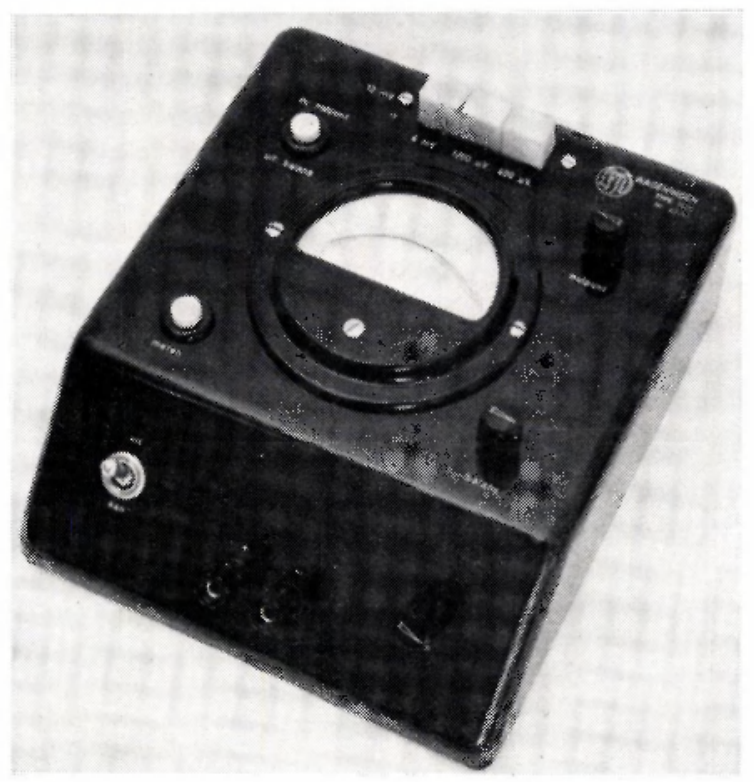


$\mathrm{Cu}-\mathrm{C}$ thermocouple, the most sensitive range corresponds to a temperature traject of approx. $2.5^{\circ} \mathrm{C}$. The input resistance is $10,000 \Omega$ in the most sensitive range.

Hence the error due to the combined resistance of the thermocouple and the reference couple, viz. $18 \Omega$, is only $0.2 \%$.

A microvoltmeter based on the chopper principle is now being developed, so that zero and balance settings can be avoided (FIG. 7). The input D.C. voltage is converted by a transistorized chopper into an A.C. voltage with a frequency of $400 \mathrm{c} / \mathrm{s}$ amplified by an A.C. amplifier and rectified by a phase-sensitive demodulator.

The input resistance will be increased, the sensitivity remaining unchanged.

FIG. 7. Chopper-stabilized microvoltmeter

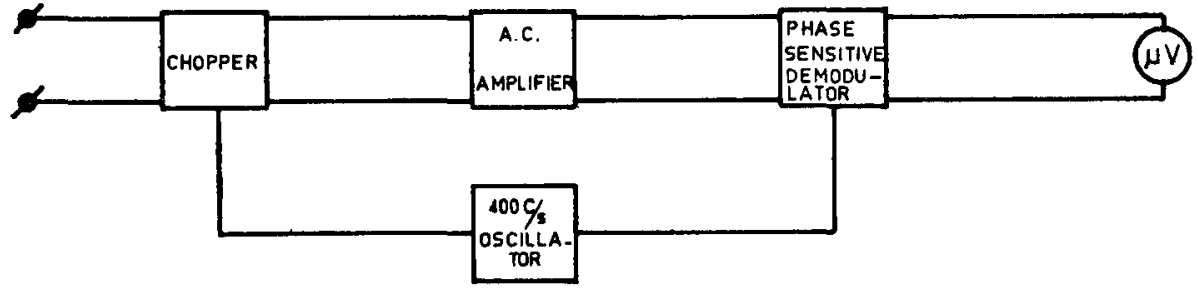

The sensitive electronic components of the apparatus are combined in small units, filled up with resin, to make the apparatus moisture- and heat-proof and thus suitable for use in the tropics.

Several electronic components must be selected carefully because they have to meet special requirements made by the circuit of the microvoltmeter. The completed circuit should be tested to obtain maximum accuracy and stability for the whole instrument.

\section{Uses}

Measurement of the thermal behaviour of a soil probe is of special interest for studying the thermal properties of a soil with respect to soil composition and arrangement of the soil components. Data obtained by such measurements can be used in microclimatology studies. A typical and up-to-date application of the method is found in the increasing use of soil heating elements, especially in horticulture.

Since the measurement leaves the soil practically undisturbed, information can be obtained for elucidating soil-water transport problems.

Hitherto we have not undertaken any studies on such similar materials as fertilizers, stored seeds, feeding materials, etc. The method would seem to be promising for determining the moisture content of such materials.

\section{R E F E R E N C E S}

DRUNen, F. C. vaN

Lab. of Soils and Fertilizers, Wageningen

LIEN, D. H.
1949 Meting van de warmtegeleiding in vloeistoffen. (Measuring thermal conductivity in liquids). Thesis. Utrecht, 1949. $71 \mathrm{pp}$. Laboratory manual course soils and fertilizers $\mathbf{A}$.

1962 Constant-current regulator speeds relay testing. Electronics. p. 48 . 
MEASUREMENT OF THERMAL CONDUCTIVITY IN SITU IN MIXED MATERIALS, E.G. SOILS

MiddlebrooK, R. D., and A. D. TAYLOR VRIES, D. A. DE

WIJK, W. R. VAN
1961 Differential amplifier with regulator achieves high stability, low drift. Electronics. p. 56.

1952 A non-stationnary method for determining thermal conductivity of soil in situ. Soil Science. 73, 83-89.

Chapter 7, Thermal properties of soils. 210-235. In: W. R. van Wijk's Physics of plant environment.

1963 Physics of plant environment. 